PREPARED FOR THE U.S. DEPARTMENT OF ENERGY, UNDER CONTRACT DE-AC02-76CH03073

PPPL-3520

PPPL-3520

UC-70

Raman Forward Scattering in Plasma Channels

by

G. Shvets and X. Li

November 2000

M

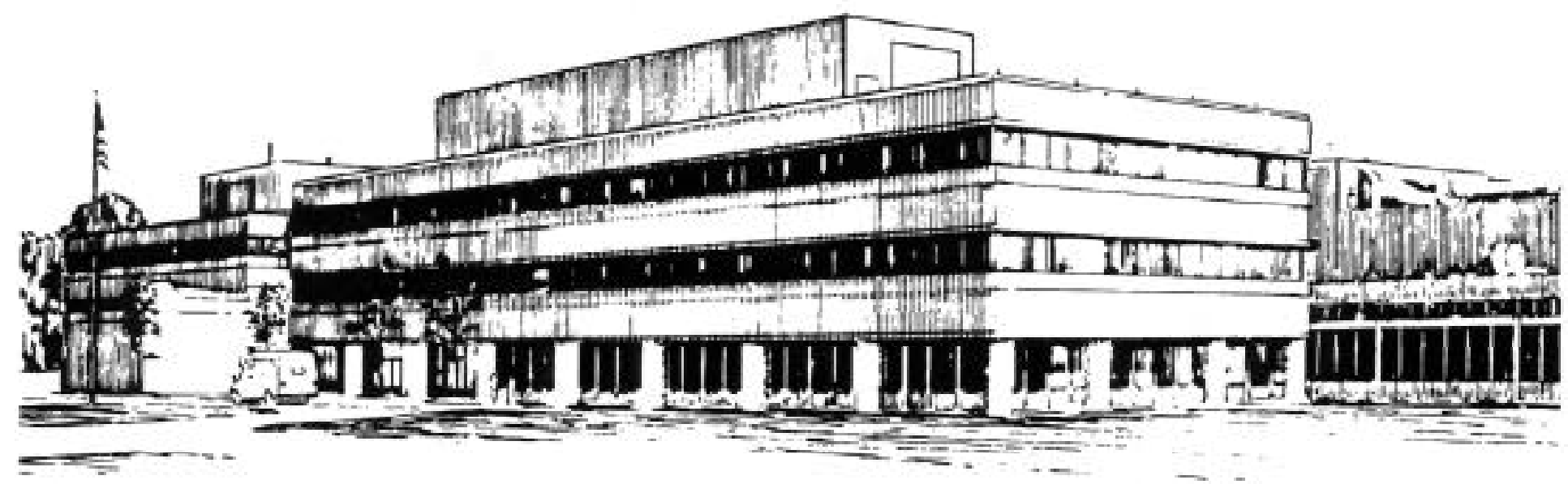

PRINCETON PLASMA PHYSICS LABORATORY PRINCETON UNIVERSITY, PRINCETON, NEW JERSEY 


\section{PPPL Reports Disclaimer}

This report was prepared as an account of work sponsored by an agency of the United States Government. Neither the United States Government nor any agency thereof, nor any of their employees, makes any warranty, express or implied, or assumes any legal liability or responsibility for the accuracy, completeness, or usefulness of any information, apparatus, product, or process disclosed, or represents that its use would not infringe privately owned rights. Reference herein to any specific commercial product, process, or service by trade name, trademark, manufacturer, or otherwise, does not necessarily constitute or imply its endorsement, recommendation, or favoring by the United States Government or any agency thereof. The views and opinions of authors expressed herein do not necessarily state or reflect those of the United States Government or any agency thereof.

\section{Availability}

This report is posted on the U.S. Department of Energy's Princeton Plasma Physics Laboratory Publications and Reports web site in Calendar Year 2000. The home page for PPPL Reports and Publications is: http://www.pppl.gov/pub_report/

DOE and DOE Contractors can obtain copies of this report from:

U.S. Department of Energy

Office of Scientific and Technical Information

DOE Technical Information Services (DTIS)

P.O. Box 62

Oak Ridge, TN 37831

Telephone: (865) 576-8401

Fax: (865) 576-5728

Email: reports@adonis.osti.gov

This report is available to the general public from:

National Technical Information Service

U.S. Department of Commerce

5285 Port Royal Road

Springfield, VA 22161

Telephone: $1-800-553-6847$ or

(703) $605-6000$

Fax: (703) 321-8547

Internet: http://www.ntis.gov/ordering.htm 


\title{
Raman Forward Scattering in Plasma Channels
}

\author{
G. Shvets and X. Li \\ Princeton Plasma Physics Laboratory, Princeton University, Princeton, NJ 08543
}

\begin{abstract}
Raman scattering instability of an intense laser pulse in a plasma channel proceeds differently than in a homogeneous plasma: the growth rate is reduced and the scaling with the laser intensity modified. These differences, significant even for shallow plasma channels, arise because of the radial shear of the plasma frequency and the existence of the weakly damped hybrid (electrostatic/electromagnetic) modes of the radially inhomogeneous plasma. The interplay of these two effects produces double-peaked spectra for the direct forward scattering in a channel.
\end{abstract}

PACS: 52.40.Nk, 41.75.Jv, 52.35.Mw, 42.65.Wi 
Stimulated Raman scattering (SRS) in the plasma is one of the fundamental and well researched parametric instabilities of intense laser pulses. The physical mechanism of the SRS in a transversely uniform plasma is well understood: the ponderomotive beatwave between the pump $\left(\omega_{0}\right)$ and its Stokes/anti-Stokes sidebands $\left(\omega_{0} \pm \omega_{p}\right)$ drive a plasma wave $\left(\omega_{p}\right)$, which then acts as a grating, scattering the pump and re-enforcing the sidebands. In this Letter we demonstrate that the SRS is strongly modified in a plasma channel, where the plasma frequency $\omega_{p}=\sqrt{4 \pi e^{2} n_{0} / m}$ is radially sheared through the radial dependence of the plasma density $n_{0}(r)$ (here $-e$ and $m$ are the electron charge and mass). From the practical standpoint, the stability of the laser propagation in channels needs to be understood as the plasma channels are increasingly used for the uninhibited by diffraction guiding of intense laser pulses over extended distances [1,2], with applications including X-ray lasers [3], inertial confinement fusion (ICF) [4], and laser-plasma accelerators [5,6].

From the physics standpoint, two novel effects modify the SRS spectrum. The first effect, plasma wave localization, is caused by the radial shear of the plasma frequency. Indeed, the low-frequency beatwave with $\omega \ll \omega_{0}$ efficiently excites the plasma wave only in the vicinity of a specific radius $r$, where $\omega_{p}(r)=\omega$. This reduces the overlap between the lasers and the plasma wave and, consequently, the SRS growth rate. The second (electromagnetic) effect occurs because the low-frequency plasma wave, which is purely electrostatic in a homogeneous plasma, acquires an electromagnetic component in a plasma channel, turning into a weakly damped quasi-mode [7,8]. The SRS growth rate is then further modified by the quasi-mode's participation.

The first effect is important whenever the SRS growth rate is smaller than the defined below variation of the plasma frequency across the channel $\Delta \omega_{p}$. In several physical situations this is indeed the case. Raman backscattering (RBS) in the plasma has recently been proposed as a technique for compressing low-intensity laser pulses [9] with the normalized vector potential $a_{0}=e A_{0} / m c^{2} \ll 1$. Since the growth rate of the RBS $\gamma_{\mathrm{rbs}}=0.5 \sqrt{\omega_{0} \omega_{p}} a_{0} \ll \omega_{p}$, even a shallow plasma channel can modify the instability. At higher laser intensities, Raman Forward Scattering (RFS) can produce energetic electrons [10] for ICF target ignition 
or high-energy physics. It's growth rate $\gamma_{\mathrm{rfs}}=a_{0} \omega_{p 1}^{2} / \sqrt{8} \omega_{0}[11,12]$ will also be modified by the plasma channel. In addition, in the case of the RFS instability, the electromagnetic effect becomes equally important. The interplay of these two effects produces a peculiar double-humped gain profile of the Raman Forward Scattering RFS in the channel, shown as a solid line in Fig.1 (b).

To examine both effects, we focus on the Raman Forward Scattering (RFS) in a singlemode flat plasma channel, in which the plasma density $n_{0}(x)$ is a function of a single transverse coordinate, and the plasma frequency varies between $\omega_{p 1}=\sqrt{\omega_{p 2}^{2}-\Delta \omega_{p}^{2} / 2}$ at $x=0$ and $\omega_{p 2}$ at $|x| \rightarrow \infty$ according to

$$
\omega_{p}^{2}=\omega_{p 2}^{2}-\frac{\Delta \omega_{p}^{2}}{2 \cosh ^{2}(x / \sigma)},
$$

The single-mode assumption enables neglecting side-scattering instabilities. In order to quantitatively describe the plasma wave localization in the channel, we neglect the selfmodulation instability (SMI) [13-15] which, undoubtedly, is also modified. Dispersion relation for a circularly polarized pump $\vec{a}_{0}=a_{0} / 2\left(\vec{e}_{x}+i \vec{e}_{y}\right) e^{i \theta_{0}}+$ c.c. with $\theta_{0}=\left(k_{0} z-\omega_{0} t\right)$ can be derived by solving the eigenvalue equation $\mathcal{L}_{0} a_{0}=\lambda_{0} a_{0}$, where $\lambda_{0}=\omega_{0}^{2} / c^{2}-k_{0}^{2}$ and

$$
\mathcal{L}_{0}=-\frac{\partial^{2}}{\partial x^{2}}+\frac{\omega_{p}^{2}(x)}{c^{2}}\left(1-\frac{\left|a_{0}^{2}\right|}{2}\right) .
$$

In deriving Eq. (2) $\left|a_{0}^{2}\right| \ll 1$ is assumed (weakly-relativistic pump), and the density depression created by the ponderomotive pressure of the laser pulse is neglected. Relativistically modified plasma density $U_{0}(x)=k_{p}^{2}\left(1-\left|a_{0}^{2}\right| / 2\right)$ plays the role of the self-consistent confining potential with a minimum at $x=0$ (here and elsewhere $k_{p}=\omega_{p} / c$ ). Assuming a shallow channel with $\Delta \omega_{p}^{2}<\omega_{p 2}^{2}$ and neglecting the second-order $\Delta \omega_{p}^{2} u_{0}^{2} / \omega_{p 2}^{2}$ term, the amplitude $u_{0}$ of a single bound (fundamental) mode $a_{0}(x)=u_{0} \psi_{0} \equiv u_{0} \cosh ^{-1}(x / \sigma)$ is found to be related to the laser spotsize through $\left(\Delta k_{p}^{2}+k_{p 2}^{2} u_{0}^{2}\right) \sigma^{2}=4$, resulting in the weaklynonlinear pump dispersion relation $\omega_{0}^{2} / c^{2}-k_{0}^{2}=k_{p 2}^{2}-\sigma^{-2}$. The confining potential is then $U_{0} \approx k_{p 2}^{2}-\left(\Delta k_{p}^{2}+k_{p 2}^{2} u_{0}^{2}\right) / 2 \cosh ^{2}(x / \sigma)$, and the eigenmodes $\psi_{q}$ of the transverse operator $\mathcal{L}_{0}$ are found by solving the eigenvalue equation in $y \equiv \tanh (x / \sigma)$ : 


$$
\frac{\partial}{\partial y}\left[\left(1-y^{2}\right) \frac{\partial \psi_{q}}{\partial y}\right]+\left[s(s+1)-\frac{\mu^{2}}{1-y^{2}}\right] \psi_{q}=0
$$

where $\psi_{q}$ 's are defined inside the $-1<y<1$ interval, $2 s(s+1)=\left(\Delta k_{p}^{2}+k_{p 2}^{2} u_{0}^{2}\right) \sigma^{2}$, and $\mu^{2}=\left(k_{p 2}^{2}-\lambda_{q}\right) \sigma^{2}$. The solutions of this equation are the associated Legendre functions $P_{s}^{\mu}(y)$ [16], and the spectrum contains $s$ discrete energy levels with $\mu^{2}>0$ (in our case $s=1$ ) and a continuum of modes with $\mu^{2}=-q^{2} \sigma^{2}<0$, where $q$ labels the continuum modes which behave $\propto \exp ( \pm i q x)$ at infinity. In the remainder of this paper we assume that focusing is primarily provided by the pre-formed channel, $k_{p 2}^{2} u_{0}^{2} \sigma^{2} \ll 4$, which is equivalent to assuming that the laser power is below the relativistic focusing threshold in the slab geometry.

We proceed by Fourier-Laplace transforming the envelope of the perturbed laser field and separating the Stokes/anti-Stokes components $\tilde{a}_{ \pm}$according to

$$
\delta \tilde{a}=\sum_{\omega, k}\left(\tilde{a}_{+} e^{i(k z-\omega t)}+\tilde{a}_{-} e^{-i\left(k z-\omega^{*} t\right)}\right)+c . c .
$$

Wave equation for for $a_{+}$(tildes are dropped for compactness) becomes $\left(\mathcal{L}_{0}-\Delta_{+}\right) a_{+}=$ $a_{0}^{2} k_{p}^{2}\left(a_{+}+a_{-}^{*}\right) / 2-k_{p}^{2}\left(\delta n / n_{0}\right) a_{0}$, where $\Delta_{ \pm}=\left(\omega_{0} \pm \omega\right)^{2} / c^{2}-\left(k_{0} \pm k\right)^{2}$, and the equation for the ponderomotively-driven density perturbation $\delta n / n_{0}$ is derived shortly. For $\omega$ close to $\omega_{p}$ (Raman process), the first term in the right-hand side (RHS) of the wave equation (due to the relativistic mass increase) is smaller than the second (due to the resonant excitation of the plasma wave). Plasma wave is driven by the intensity modulation of the laser field given by $|a|^{2}=a_{0}^{2}+a_{0}\left(a_{+}+a_{-}^{*}\right) e^{i(k z-\omega t)}+$ c. c., where the perturbed laser field is expanded as $a_{ \pm}=a_{ \pm}^{(0)} \psi_{0}(x)+\sum a_{ \pm}^{(q)} \psi_{q}(x)$. Inserting Eq. (4) into the wave equation, multiplying its both sides by $\psi_{0}(x)$, and integrating over $x$, obtain (using the orthogonality condition $\left.<\psi_{0}, \psi_{q}>=0,<\psi_{q}, \psi_{q^{\prime}}>=\delta_{q q^{\prime}}\right)$

$$
\left(\lambda_{0}-\Delta_{+}\right) a_{+}^{(0)}=-u_{0} \frac{\left\langle\delta k_{p}^{2}, \psi_{0}^{2}\right\rangle}{\left\langle\psi_{0}, \psi_{0}\right\rangle}+\sum_{q} N_{q},
$$

where $\delta k_{p}^{2}=k_{p}^{2} \delta n / n_{0}$ is proportional to the density variation due to the perturbation of the bound mode $a_{ \pm}^{(0)}$, and $N_{q}=u_{0}\left\langle\delta k_{p}^{2(q)}, \psi_{0}^{2}\right\rangle / 2\left\langle\psi_{0}, \psi_{0}\right\rangle$, where $\delta k_{p}^{2(q)}$, defined by analogy with $\delta k_{p}^{2}$, is the partial contribution of the continuum mode $q$ to the RHS of the wave equation. 
Continuum and bound modes are treated separately because we assume that only the bound mode becomes unstable. The unbounded modes are not independently unstable because they describe diffracting radiation which does not overlap with the pump $a_{0}$ for a sufficient time to get significantly amplified by the small-angle Raman scattering [17]. Some nonlinear coupling to the continuum modes does, however, take place: they are driven by the exponentially growing bound mode $a_{ \pm}^{(0)}[18]$. The equation for $a_{+}^{(q)}$ corresponding to Eq. (5) is given by $\left(\lambda_{q}-\Delta_{+}^{(q)}\right) a_{+}^{(q)}=-u_{0}\left\langle\delta k_{p}^{2}, \psi_{0} \psi_{q}\right\rangle$, where the $q-q^{\prime}$ coupling between the continuum modes is dropped, and $\Delta_{+}^{(q)}$ is the same as $\Delta_{+}$, except that $\omega$ and $k$ correspond to the continuum mode $q$.

If the growth rate $\gamma$ is small $\left(2 \gamma \omega_{0} / c^{2}<\lambda_{q}-\lambda_{0}\right.$, or the growth length is longer than the Rayleigh length), then $a_{ \pm}^{(q)}$ adiabatically follows the bound mode. The continuum modes can, in effect, be eliminated by assuming that $\Delta_{+}^{(q)}=\Delta_{+}^{(0)} \approx \lambda_{0}$, resulting in $\left(\lambda_{q}-\lambda_{0}\right) a_{+}^{(q)}=-u_{0}\left\langle\delta k_{p}^{2}, \psi_{0} \psi_{q}\right\rangle$. This expression for $a_{+}^{(q)}$ can be used for calculating $\delta k_{p}^{2(q)}$ and inserted into $N_{q}$, resulting in the generalized one-dimensional dispersion relation for the bound mode which accounts for the coupling to the continuum modes. In particular, balancing $\sum_{q} N_{q}$ against the first terms in the RHS of Eq. (5) provides the rate of the self-modulation instability in the paraxial approximation [13-15] which is neglected in this Letter in order to elucidate the modification of the RFS by the plasma wave localization.

Neglecting the continuum modes' correction $\sum N_{q}$ (which is proportional to $a_{0}^{4}$ ) in Eq. (5) results in the familiar from Refs. [11,12] expression

$$
\frac{D_{+} D_{-}}{D_{+}+D_{-}}\left(a_{+}^{(0)}+a_{-}^{*(0)}\right)=u_{0} \frac{\left\langle\delta k_{p}^{2}, \psi_{0}^{2}\right\rangle}{\left\langle\psi_{0}, \psi_{0}\right\rangle}
$$

where $D_{ \pm}= \pm 2\left(\omega_{0} \omega / c^{2}-k_{0} k\right)+\left(\omega^{2} / c^{2}-k^{2}\right)$. The commonly used [15,17,19] simplified equation $\left(\partial_{t}^{2}+\omega_{p 1}^{2}\right) \delta n / n=\nabla^{2}|a|^{2} / 2 c^{2}$ for the density perturbation does not correctly describe the plasma wave localization because it assumes that the plasma wave has a single response frequency (SRF) $\omega_{p 1}$. In reality, there is a continuum of plasma frequencies between $\omega_{p 1}$ and $\omega_{p 2}$, and the phase-mixing of various plasma waves from this continuum leads to their spatial localization. More rigorously, $\delta k_{p}^{2}$ in a channel is given by [8] 


$$
\delta k_{p}^{2}=\frac{1}{2}\left[\frac{\omega_{p}^{2}(x)}{\omega_{p}^{2}-\omega^{2}} \nabla^{2}+\frac{\epsilon^{\prime}}{\epsilon^{2}} \frac{\partial}{\partial x}\right] b-\frac{\epsilon^{\prime}}{\epsilon^{2}} \frac{e B_{y}}{m c^{2}},
$$

where $\epsilon(x, \omega)=1-\omega_{p}^{2}(x) / \omega^{2}$, the prime denotes a derivative with respect to $x, b \equiv a_{0}\left(a_{+}+\right.$ $a_{-}^{*}$, and $\tilde{B}=e_{y} B_{y}$ is the magnetic field which satisfies

$$
B_{y}^{\prime \prime}-\frac{\epsilon^{\prime}}{\epsilon} B_{y}^{\prime}-\frac{\omega_{p}^{2}(x)+k^{2} c^{2}-\omega^{2}}{c^{2}} B_{y}=-\frac{m \omega^{2} \epsilon^{\prime} b}{2 e \epsilon} .
$$

Equation (7) is obtained from Eq. (3) of the Ref. [8] by taking the divergence of $\vec{E}$. From Eq. (7), the density perturbation can be broken up into two parts: $\delta k_{p}^{2}=\delta k_{p}^{2(L)}+\delta k_{p}^{2(B)}$, where the first contribution (in square brackets) is locally-driven, i. e. $\delta k_{p}^{2(L)}(x)$ is determined by the ponderomotive force at the same $x$. The second contribution $\delta k_{p}^{2(B)}$ is related to the magnetic field $B_{y}$ and is manifestly non-local.

For the Raman backscattering (RBS), $c k \gg \omega$, and the plasma wave is predominantly electrostatic. In this case $\delta k_{p}^{2(L)}$ is sufficient for calculating the instability growth rate. For the RFS, $\omega \approx c k$, and the electromagnetic part of the plasma wake, characterized by $B_{y}$, can be as important as the electrostatic one. The localized contribution can be calculated analytically. We do this first, and return to the numerical estimation of $\delta k_{p}^{2(B)}$ later on. Substituting $\delta k_{p}^{2(L)}$ into Eq. (6), obtain the dispersion relation $D_{+} D_{-} /\left(D_{+}+D_{-}\right)=u_{0}^{2} \tilde{Q}$, where

$$
\tilde{Q}=\int_{-1}^{1} d y\left(1-y^{2}\right)\left[\frac{y^{2}}{\sigma^{2}}+\frac{\omega^{2}}{4 c^{2}}\right] \frac{\omega_{p 1}^{2}+\Delta \omega_{p}^{2} y^{2} / 2}{\left(\omega^{2}-\omega_{p 1}^{2}\right)-\Delta \omega_{p}^{2} y^{2} / 2}
$$

The plasma response function $\tilde{Q}(\omega)$ can be evaluated analytically; for a broad shallow channel $\omega^{2} \sigma^{2} / c^{2} \gg 1$ the expression for $\tilde{Q}$ is particularly simple, yielding

$$
\begin{aligned}
& \frac{D_{+} D_{-}}{D_{+}+D_{-}}=\frac{\omega^{2}}{4 c^{2}} \frac{\omega_{p 1}^{2} u_{0}^{2}}{\omega^{2}-\omega_{p 1}^{2}}\left[\frac{2}{B^{2}}\left(1-\frac{2 C^{2}}{3}+\frac{C^{2}}{B^{2}}\right)\right. \\
& \left.-\frac{\left(B^{2}+C^{2}\right)\left(1-B^{2}\right)}{B^{5}} \ln \left(\frac{1+B}{1-B}\right)\right],
\end{aligned}
$$

where $C^{2}=\Delta \omega_{p}^{2} / 2 \omega_{p 1}^{2}$ and $B^{2}=\Delta \omega_{p}^{2} / 2\left(\omega^{2}-\omega_{p 1}^{2}\right)$. Equation (10) is the first, to our knowledge, closed-form dispersion relation for the SRS instability in a plasma channel which correctly describes the plasma wave excitation and localization; it is valid for both the 
forward and backward Raman scattering. For the previously studied RFS in very shallow [15] and hollow [20] channels plasma wave localization was not an issue.

The almost-homogeneous plasma limit is recovered by expanding Eq. (10) in the powers of small $B$ and $C: \tilde{Q} \approx k_{p 1}^{2} \omega^{2} / 3\left(\omega^{2}-\omega_{p 1}^{2}\right)$. In the time domain, $Q(t)$ describes an undamped channel-averaged plasma oscillation $Q \propto \sin \omega_{p 1} t$ : just as expected from the simplified SRF equation. For $\omega=\omega_{p 1}+i \gamma$ and $k=\omega_{p 1} / v_{g 0}$ the peak temporal growth rate $\gamma_{\text {hom }} \approx$ $u_{0} \omega_{p 1}^{2} / \sqrt{6} \omega_{0}$ (where we used $D_{+} D_{-} /\left(D_{+}+D_{-}\right) \approx-i \omega_{0}^{2} \gamma / \omega_{p 1} c^{2}$, and $v_{g 0}=c^{2} k_{0} / \omega_{0}$ is the group velocity of the pump). This growth rate is almost identical to $\gamma_{\mathrm{rfs}}$ in the homogeneous plasma. The above estimate of $\gamma$ relies on $\left|B^{2}\right| \ll 1$, or $u_{0}>(\sqrt{6} / 4) \Delta \omega_{p}^{2} \omega_{0} / \omega_{p 1}^{3}$. Even for a very shallow $\Delta \omega_{p}^{2} / \omega_{p 1}^{2}=0.2(10 \%$ density depression $)$ channel with $\omega_{0} / \omega_{p 1}=10$, the homogeneous plasma result is valid for $u_{0}>1.2$. Physically, this means that the plasma wave localization can only be neglected if the RFS rate is high, $\gamma>\Delta \omega_{p}^{2} / 2 \omega_{p 1}$. More accurately, $\gamma_{\text {hom }}$ is derived using the exact expression (9) for $\tilde{Q}$ :

$$
\gamma_{\mathrm{hom}}=\frac{u_{0} \omega_{p 1}^{2}}{\sqrt{6} \omega_{0}} \sqrt{1+\frac{1}{10} \frac{\Delta \omega_{p}^{2}}{\omega_{p 1}^{2}}+\frac{4}{5} \frac{c^{2}}{\omega_{p 1}^{2} \sigma^{2}}+\frac{6}{35} \frac{\Delta \omega_{p}^{2}}{\omega_{p 1}^{2}} \frac{c^{2}}{\omega_{p 1}^{2} \sigma^{2}}} .
$$

The importance of the localization is measured by the dimensionless parameter $\eta=$ $\Delta \omega_{p}^{2} \omega_{0} / u_{0} \omega_{p 1}^{3}$ which is proportional to the ratio of the channel depth and the growth rate. For $\eta>1$, the $\left|B^{2}\right| \gg 1$ limit of $\tilde{Q}$ yields

$$
\tilde{Q}=\frac{\omega^{2}}{c^{2}} \frac{\omega_{p 1}^{2}}{\Delta \omega_{p}^{2}}\left[\frac{-i \pi \Delta \omega_{p}}{\sqrt{8}\left(\omega^{2}-\omega_{p 1}^{2}\right)^{1 / 2}}+\left(2-\frac{\Delta \omega_{p}^{2}}{3 \omega_{p 1}^{2}}\right)\right]
$$

The physical meaning of $\left|B^{2}\right| \gg 1$ is that a plasma wave has a transverse extent $\delta x \approx$ $\sigma /|B|$, i. e. it is more localized than the driving beatwave. Localization reduces the overlap between the plasma wave and the beatwave, affecting the growth rate. In the time domain, localization manifests itself in the algebraic decay of the plasma response for $t \rightarrow \infty: Q \propto$ $\sin \left(\omega_{p 1} t\right) / \sqrt{t}$.

The analytic estimates of the growth rate are obtained in two limits: gain-dominated and dispersion-dominated. In the gain-dominated case $\gamma>\omega_{p 1}^{3} / 2 \omega_{0}^{2}$ (which corresponds to $\left.u_{0}^{2}>0.8 \Delta \omega_{p} / \omega_{0}\right)$ obtain 


$$
\omega-\omega_{p 1}=e^{i \pi / 3}\left(\frac{u_{0} \omega_{p 1}^{2}}{\omega_{0}}\right)\left[\frac{\pi^{2}}{16 \eta}\right]^{1 / 3} .
$$

From Eq. (12), note the unusual scaling of the growth rate with the laser intensity $\gamma \propto$ $I_{0}^{2 / 3}$. For comparison, $\gamma_{\text {hom }} \propto I_{0}^{1 / 2}$ for the resonant Raman instabilities in the homogeneous plasma, but $\gamma \propto I_{0}$ for the non-resonant SRS in glass fibers [21]. That the intensity scaling of the SRS growth rate in a plasma channel falls between those in the homogeneous plasma and in the glass is explained by the time dependence of the response functions $Q$. The corresponding $Q$ 's of the homogeneous plasma, plasma channel, and the glass fiber decay progressively faster: hence the correspondingly stronger scalings of the gain with the laser intensity.

In the opposite dispersion-dominated $\left(u_{0}^{2}<0.8 \Delta \omega_{p} / \omega_{0}\right)$ limit, the growth rate can also be evaluated analytically. A straightforward (although cumbersome) calculation yields $\gamma_{d} \approx$ $\gamma_{\mathrm{hom}} \sqrt{6 \omega_{p 1} / \omega_{0} \Delta \omega_{p}}$. The solid line in Fig. 1(a) is the dependence of the peak growth rate on $u_{0}$, obtained by solving the dispersion relation with the exact $\tilde{Q}$ for the plasma channel with a $40 \%$ density depression. The $\gamma_{\mathrm{hom}}$ (dotted line) over-estimates the growth rate for all values of $u_{0}$. The dashed-dotted and dashed lines are the growth rates in the dispersion and gain-dominated regimes, respectively. Consistently with the assumptions used in deriving Eq. (12) and $\gamma_{d}$, the growth rate is better approximated by the former for large $u_{0}$ and by the latter for small $u_{0}$. The RFS spectrum calculated from the exact $\tilde{Q}$ is shown in Fig. 1(b) (dashed line) for $u_{0}=0.35$.

We find that the RFS growth rate and spectrum are significantly modified after the magnetic field contribution $\delta k_{p}^{2(B)}$ is added to the locally-driven density perturbation $\delta k_{p}^{2(L)}$. The numerically calculated overlap integral

$$
u_{0} \frac{\left\langle\delta k_{p}^{2(B)}, \psi_{0}^{2}\right\rangle}{\left\langle\psi_{0}, \psi_{0}\right\rangle}=\frac{u_{0}^{2}}{2 c^{2}} \frac{\omega^{4} \Delta \omega_{p}^{4}}{\left(\omega^{2}-\omega_{p 1}^{2}\right)^{3}} \int_{0}^{1} d y \frac{y\left(1-y^{2}\right) H_{1}}{\left(1-B^{2} y^{2}\right)^{2}}
$$

contains the normalized magnetic field $H_{1}$ satisfying

$$
\begin{aligned}
& \frac{\partial}{\partial y}\left[\frac{1-y^{2}}{1-B^{2} y^{2}} \frac{\partial H_{1}}{\partial y}\right]+\sigma^{2}\left(\frac{\Delta \omega_{p}^{2}}{2 c^{2}}-\frac{k_{p 2}^{2}}{1-y^{2}}\right) \times \\
& \times \frac{H_{1}}{1-B^{2} y^{2}}=-\frac{y\left(1-y^{2}\right)}{\left(1-B^{2} y^{2}\right)^{2}},
\end{aligned}
$$


with $H_{1}(y=0)=H_{1}(y=1)=0$. Equation (13) follows from Eq. (8) by setting $\omega=k c$; its LHS describes the collisionlessly-damped global quasi-mode [8] of the plasma channel. Boundary conditions for $H_{1}$ are satisfied for a single value of $B$ which determine the frequency and damping rate of the quasi-mode. For example, for a plasma channel with a $40 \%$ density depression these are equal to $\omega_{Q}=0.82 \omega_{p 2}$ and $\gamma_{Q}=0.045 \omega_{p 2}$. Qualitatively, damping of the hybrid wave in a smooth plasma channel is related to the phase-mixing of the plasma oscillations which support this wave at location $x$. Since these supporting plasma fluid elements are within $\Delta x=k_{p}^{-1}$ of $x$, the difference between the local oscillation frequencies $\omega_{p}$ leads to phase-mixing and eventual damping of the wave [8].

The total peak growth rate $\gamma$ (with $\delta k_{p}^{2(B)}$ added), marked by squares in Fig. 1(a), is increased by the addition of the non-electrostatic contribution. That the total $\gamma$ is rather close to $\gamma_{\text {hom }}$ appears to be a coincidence. The modification of the RFS spectrum [Fig. 1(b)] by the addition of $\delta k_{p}^{2(B)}$ is observed as a distinctive broad amplification band peaked at $\omega_{r}=0.91 \omega_{p 2}$. It is slightly shifted from the quasi-mode frequency $\omega_{Q}=0.82 \omega_{p 2}$. The double-humped RFS spectrum is the result of the interplay between the two novel effects which appear during the SRS in a plasma channel: (a) plasma wave localization caused by the phase-mixing of plasma oscillations, and (b) non-electrostatic nature of the plasma waves in a channel. We speculate that these newly uncovered phenomena might also affect other laser instabilities in plasma channels, such as the self-modulation instability. 


\section{REFERENCES}

[1] C. Durfee and H. Milchberg, Phys. Rev. Lett. 71, 2409 (1993).

[2] A. Ziggler, Y. Ehrlich, C. Cohen, J. Krall, and P. Sprangle, J. Opt. Soc. Am. B 13, 68 $(1996)$.

[3] C. Clark, M. G. Littman, R. Miles, T. J. McIlrath, C. H. Skinner, and S. Suckewer, J. Opt. Soc. Am. B 3, 371 (1986).

[4] M. Tabak, J. Hammer, M. E. Glinsky, W. L. Kruer, S. C. Wilks, J. Woodworth, E. M. Campbell, and M. D. Perry, Phys. Plasmas 1, 1626 (1994).

[5] T. Tajima and J. M. Dawson, Phys. Rev. Lett. 43, 267 (1979).

[6] E. Esarey, P. Sprangle, J. Krall, and A. Ting, IEEE Trans. Plasma Science 24, 252 (1996), and references therein.

[7] G. Shvets, J. S. Wurtele, T. C. Chiou, and T. Katsouleas, IEEE Trans. Plasma Sci. 24, $351(1996)$.

[8] G. Shvets and X. Li, Phys. Plasmas 6, 591 (1999).

[9] V. M. Malkin, G. Shvets, and N. J. Fisch, Phys. Rev. Lett. 82, 4448 (1999); V. M. Malkin, G. Shvets, and N. J. Fisch, Phys Plasmas 7, 1020 (2000).

[10] A. Modena, A. E. Dangor, Z. Najmudin, C. E. Clayton, K. Marsh, C. Joshi, V. Malka, C. B. Darrow, C. Danson, D. Neely, and F. N. Walsh, Nature 377, 606 (1995).

[11] W. L. Kruer, The Physics of Laser Plasma Interactions (Addison-Wesley, Reading, MA, 1988) p. 78.

[12] C. J. McKinstrie and R. Bingham, Phys. Fluids B 4, 2626 (1992); W. B. Mori, C. D. Decker, D. E. Hinkel, and T. Katsouleas, Phys. Rev. Lett. 72, 1482 (1994).

[13] E. Esarey, J. Krall, and P. Sprangle, Phys. Rev. Lett. 72, 2887 (1994). 
[14] N. E. Andreev, V. I. Kirsanov, and L. M. Gorbunov, Phys. Plasmas 2, 2573 (1995).

[15] E. Esarey, C. B. Schroeder, B. A. Shadwick, J. S. Wurtele, and W. P. Leemans, Phys. Rev. Lett. 84, 3081 (2000).

[16] I. S. Gradshteyn and I. M. Ryzhik, Table of Integrals, Series, and Products (Academic Press, New York, 1965), p. 804.

[17] T. M. Antonsen, Jr.,and P. Mora, Phys. Rev. Lett. 69, 2204 (1992); T. M. Antonsen, Jr., and P. Mora, Phys. Fluids B 5, 1440 (1993).

[18] G. Tempea and T. Brabec, Opt. Lett. 23, 762 (1998).

[19] T. M. Antonsen, Jr. and P. Mora, Phys. Rev. Lett. 744, 4440 (1995).

[20] T. C. Chiou, T. Katsouleas, W. B. Mori, and G. Shvets, Proc. Adv. Accel. Concepts Workshop, Lake Tahoe, CA, October 1996, ed. S. Chattopadhyay, AIP Conf. Proc., 398, 357 (1997).

[21] G. P. Agarwal, Nonlinear Fiber Optics (Academic Press, 2-nd edition, New York, 1998) p. 316 . 


\section{FIGURES}

FIG. 1. (a) Peak growth rate $\gamma$ of RFS near the on-axis plasma frequency $\omega_{p 1}$ as a function of normalized laser amplitude $u_{0}$. Growth rate is numerically calculated from: dispersion relation $D_{+} D_{-} /\left(D_{+}+D_{-}\right)=u_{0}^{2} Q($ solid line $)$; homogeneous plasma estimate $\gamma=\gamma_{\text {hom }}($ dotted line); Eq. (12) in gain-dominated regime (dashed line); $\gamma=\gamma_{d}$ in dispersion-dominated regime

(dot-dashed line), and with the addition of $\delta k_{p}^{2(B)}$ (squares). (b) Instability spectrum for $u_{0}=0.35$ with (solid line) and without (dashed line) non-local density perturbation due to magnetic field. Channel parameters: $\omega_{p 1} / \omega_{p 2}=0.775$ and $\omega_{0} / \omega_{p 2}=10$. 
a

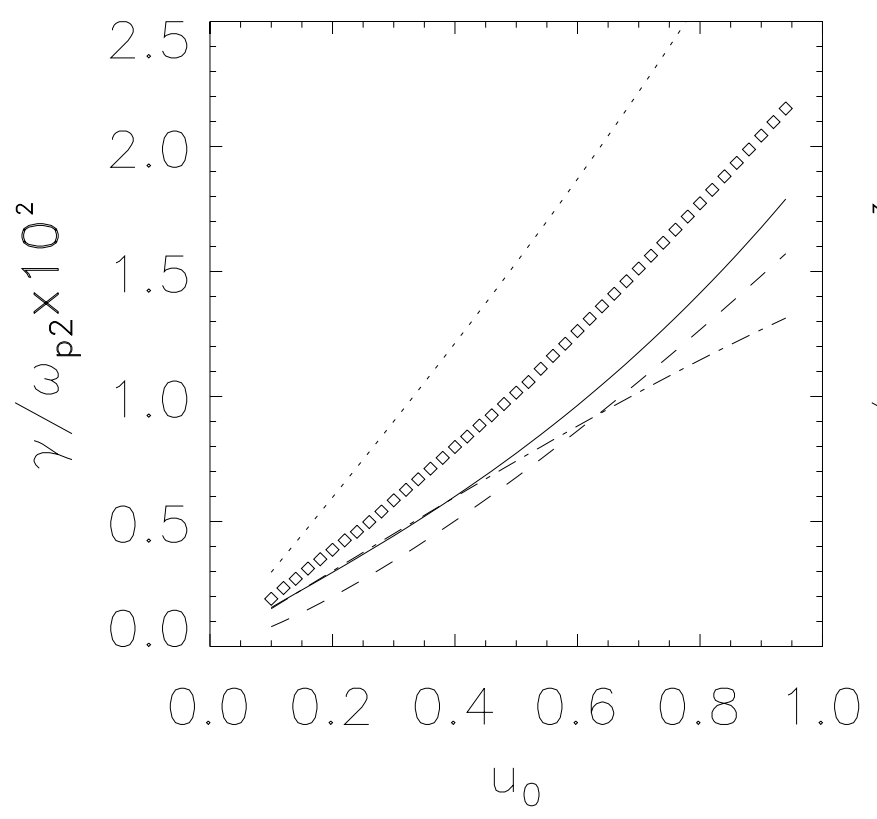

b

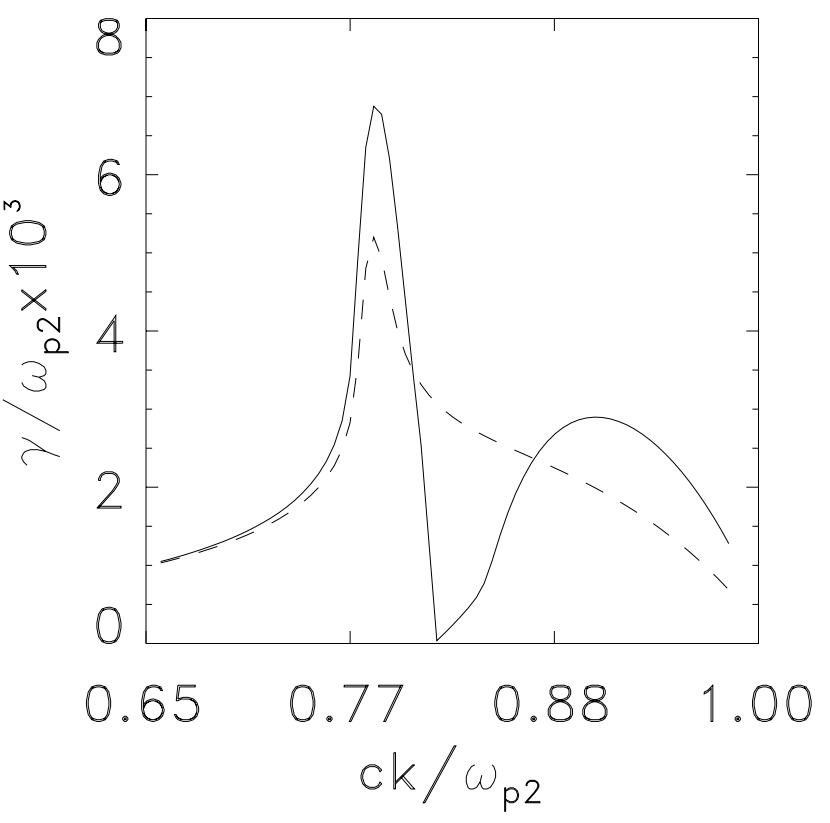


The Princeton Plasma Physics Laboratory is operated by Princeton University under contract with the U.S. Department of Energy.

\author{
Information Services \\ Princeton Plasma Physics Laboratory \\ P.O. Box 451 \\ Princeton, NJ 08543
}

Phone: 609-243-2750

Fax: 609-243-2751

e-mail: pppl_info@pppl.gov

Internet Address: http://www.pppl.gov 